\title{
Energy optimization in multiuser quantized feedback systems
}

\author{
Saud Althunibat ${ }^{1 *}$, Nizar Zorba ${ }^{2}$, Fabrizio Granelli and Christos Verikoukis ${ }^{3}$
}

\begin{abstract}
This article explores the effects of quantization of feedback information on energy consumption in multiuser wireless communication systems. In order to optimize the energy consumption of the system, the article concentrates on the amount of transmit energy, the additional energy due to quantization and the probability of power outage. Closed form expressions for such parameters are obtained, where the impact of the number of quantization bits is explicitly outlined. An optimization problem is then formulated to find the optimum number of quantization bits able to minimize the consumption in the energy resources. Simulations demonstrate the good results obtainable with the presented optimization strategy, and provide effective validation of the analytic solution presented in the article.
\end{abstract}

\section{Introduction}

Given the increased diffusion and usage of wireless and mobile networks, energy efficiency represents an open issue of paramount importance in order to support scalability and sustainability of wireless networks. Wireless network interfaces consume significant amount of energy, especially in the case of cellular base stations (BS). Indeed, the energy request for the infrastructure of wireless operators represents a large portion of the overall network costs [1]. As a consequence, decreasing the consumed energy in BSs would reduce the running costs of the whole communication infrastructure.

Channel state information (CSI) at the transmitter side is required to enable efficient resource management. Such information is obtained through a feedback process: each user periodically measures the CSI, and informs the transmitter about it. The performance in resource management by using such feedback process highly depends on the method used to represent the feedback values: where increasing the feedback values for channel state estimation would enhance the efficiency of resource management [2].

The most common approach to feedback the CSI is through the feedback quantization method, where the wide (sometimes infinite) continuous range of the CSI values is represented by finite discrete values [3], thus

*Correspondence: althunibat@disi.unitn.it

1 University of Trento, Trento, Italy

Full list of author information is available at the end of the article introducing a quantization error [4]. In multiuser systems, the users quantize their CSI, represented by the signal-to-noise ratio (SNR), into a number of bits, and send them back to the BS. As the quantized version of the SNR is represented by from a finite and low number of values, then the corresponding loss of information will negatively impact on the achieved data rate and the consumed energy, especially in cases where dynamic resource allocation is employed.

An increase to the amount of the feedback bits better matches the quantized CSI and the actual (non-quantized) CSI values [5], with a corresponding improvement in the resources distribution mechanism. On the other hand, feedback quantization is a required step in order to limit the number of bits associated to CSI feedback and avoid a potential source of inefficiency in the system. The impact of the feedback load is larger in multiuser systems, as the feedback bits are multiples of the number of active users in the cell [2]. Based on the above, an optimization to find the best amount of feedback bits is required, in order to optimize both the system performance and the energy consumption.

This article analyzes the effects of feedback quantization on energy resources and performance metrics. To this goal, the transmit energy, additional energy due to quantization, feedback energy, and the power outage probability are all derived in closed form expressions, with all the relevant parameters affecting them. Using such values, an

\section{Springer}


optimization problem for defining the appropriate number of feedback bits to increase the energy efficiency of the system is formulated.

Previous work as [6] analyzes the effect of quantization on the throughput of constant rate transmission, and it concludes that few quantization bits are required to achieve a throughput slightly lower than in the nonquantized case. However, the impact on energy consumption is not considered, while time and energy consumed during the feedback process are neglected. In [7], an optimum quantization scheme is derived for opportunistic beamforming systems, which maximizes the average system throughput while ensuring fairness among users. In [8], mathematical expressions for the rate outage probability and the capacity loss due to quantization are derived for a transmission system with finite rate feedback; while, in [9], a new quantization scheme is developed to maximize the overall throughput and minimize the scheduling error in multiuser systems. A convex optimization problem is formulated and solved in $[10,11]$, in order to find the optimal number of quantization bits that minimizes the reconstruction error in wireless sensor networks, where the constraint in [10] is the energy per sensor and in [11] is the total consumed energy. In [12], a joint channel quantization and resource allocation schemes for singleuser OFDM systems, which use limited-rate feedback, are obtained by solving an optimization problem that maximizes average ergodic rate subject to average power and bit error rate constraints. The effects of the quantization noise on energy measures, for cooperative spectrum sensing in cognitive radio networks, is investigated in [13], and a method to compensate these effects based on a new soft metric at the fusion center is proposed. The asymptotic performance of multiple antenna channels where the transmitter has finite bit CSI is analyzed in [14], where it is demonstrated that channel feedback can fundamentally change the system behavior. To the best of the authors' knowledge, there are no previous contributions on the energy efficiency for feedback quantization in opportunistic scheduling systems.

The main contributions of this article can be summarized as follows:

- Obtaining the probability density function (PDF) of the serving SNR in opportunistic scheduling in quantized feedback systems.

- Deriving closed form expressions for the transmit energy of quantized and non-quantized feedback systems, the additional energy due to quantization, and the power outage probability.

The remainder of this article is organized as follows: Section 2 describes the system model, while Section 3 discusses the effects of quantization and derives the closed form expressions, in order to formulate and solve the optimization problem to find the best amount of feedback bits that minimizes the consumed energy. Section 4 provides the numerical results and Section 5 draws conclusions and outlines future work on the subject.

\section{System model}

We focus on the downlink ${ }^{\mathrm{a}}$ channel where $N$ receivers, each one of them equipped with a single receiving antenna, are being served by a transmitter at the BS also provided with a single transmitting antenna. A channel $h(t)$ is considered between each of the users and the BS where a quasi static block fading model is assumed. The model remains constant through the coherence time, and independently changes between consecutive time intervals with independent and identically distributed (i.i.d.) complex Gaussian entries $\sim \mathcal{C N}(0,1)$. Let $s_{i}(t)$ denote the uncorrelated data symbol to the $i$ th user with $E\left\{\left|s_{i}\right|^{2}\right\}=1$, then the received signal $y_{i}(t)$ is given by

$$
y_{i}(t)=h_{i}(t) s_{i}(t)+z_{i}(t)
$$

where $z_{i}(t)$ is an additive i.i.d. complex noise component with zero mean and $E\left\{\left|z_{i}\right|^{2}\right\}=\sigma^{2}$.

At the beginning of each scheduling time, the transmitter broadcasts the training (i.e., preamble) sequence to all users, which allows each user to calculate its CSI, and decides to feedback partial CSI information, which in our case will be the SNR value. In order to feedback the SNR value, it must first be converted to a digital signal, so that each user applies the scalar quantization technique [3] that divides the whole possible SNR range in $M$ intervals, separated by $(M+1)$ thresholds $\left[0, \gamma_{1}, \gamma_{2}, \ldots, \gamma_{M-1}, \infty\right]$, so that $\left(B=\log _{2}(M)\right)$ bits are required to represent any SNR value. This uniform quantization is used since it does not require any prior information about the SNR distribution and number of users. After that, the resulting $B$ bits are fed back from each user to the BS. Certainly, some error is introduced, as the actual SNR value is approximated by the quantized SNR version. The time spent in feedback process $\left(T_{f}\right)$ is given as follows:

$$
T_{f}=\frac{N B}{D_{f}}
$$

where $D_{f}$ is the feedback data rate in bits/s. The rest of the frame, denoted as $T_{t}$, is dedicated for data transmission, and it is given as:

$$
T_{t}=T-\frac{N B}{D_{f}}
$$

where $T$ is the total frame length.

At the BS, after all the quantized SNR values are received from all users, an opportunistic scheduling technique [15-17] is employed to choose the user with best channel characteristics (i.e., maximum SNR). Therefore, the user 
with best SNR is scheduled, but due to the quantization error, more than one user may appear within the same SNR interval (i.e., the BS understands that they have the same SNR value), and hence, the BS is forced to randomly select one of them, which may yield to an erroneous selection, as the user with the best actual SNR is not selected [9]. Following commercial systems, time separation is accomplished in the feedback channel, so that the feedback is not simultaneous over all the users.

Once a user is selected, the transmit power has to be defined to meet the quality of service (QoS) demand given in terms of a minimum SNR value. If the measured SNR value is $(x)$, then the required transmit power to achieve a QoS demand $(q)$ is $\left(\frac{q}{x}\right)$. In contrast, in quantized-feedback systems, as the exact value of the measured SNR is unknown at the BS, the worst case approach is followed to allocate the transmit power [18]. The worst case approach implies that as the BS just knows the interval $\left[\gamma_{i}, \gamma_{i+1}\right]$ where the measured SNR is the lower threshold of the interval $\left(\gamma_{i}\right)$ is used to calculate the transmit power. Therefore, the transmit power in quantized feedback systems equals to $\left(\frac{q}{\gamma_{i}}\right)$.

Notice that the power allocation based on quantized feedback yields a larger amount of transmit energy, a parameter that must be optimized due to the large drawbacks of increasing the amount of transmit energy to the system, the customer and the environment ${ }^{\mathrm{b}}$.

\section{Quantization effects on the consumed energy}

To reach the optimum performance of the opportunistic scheduling, the SNR for all users must be available at the $\mathrm{BS}$, but, as we previously discussed, in practical systems the SNR values are quantized inducing a quantization error which affects the performance of the opportunistic scheduler. In this section, we concentrate on the system energy resource, and present the effects of the quantization process on the required transmit energy, additional energy, and power outage probability. After that, we formulate an optimization problem to minimize the overall energy consumption.

\subsection{Transmit energy}

It is proven that the opportunistic scheduling achieves the highest possible data rate among all other scheduling algorithms [15], leading to the minimum amount of required power to satisfy the QoS demand for the customers.

In non-quantized feedback systems, the opportunistic scheduling works perfectly and the scheduled SNR will be the maximum SNR among all users, so the average transmit power can be given as:

$$
\bar{P}_{t, n q}=\int_{0}^{\infty} \frac{q}{x} f_{\max }(x) \cdot d x
$$

where $\left(f_{\max }\right)$ is the PDF of the maximum SNR which represents the serving SNR in the non-quantized feedback system, and is given by

$$
f_{\max }(x)=N(F(x))^{N-1} f(x)
$$

where $f(x)$ is as exponential PDF that is defined as:

$$
f(x)=\sigma^{2} e^{-\left(x \sigma^{2}\right)}
$$

and $F(x)$ is the exponential cumulative distribution function which is defined as:

$$
F(x)=1-e^{-\left(x \sigma^{2}\right)}
$$

We solve Equation (4) in a closed form expression as follows (refer to Appendix for the complete derivation)

$$
\bar{P}_{t, n q}=-N \sigma^{2} q \sum_{n=0}^{N-1}\left(\begin{array}{c}
N-1 \\
n
\end{array}\right)(-1)^{n}\left(\ln \left(\sigma^{2}(n+1)\right)\right)
$$

To obtain the transmit energy in non-quantizedfeedback systems, we multiply $\bar{P}_{t, n q}$ by the transmission time $T_{t}$ as follows:

$$
E_{t, n q}=\bar{P}_{t, n q}\left(T-\frac{N B}{D_{f}}\right)
$$

In quantized-feedback systems, the scheduled SNR is not always the maximum, as explained above, so the PDF of the scheduled SNR will change as follows:

$$
f_{Q}(x)=\sum_{n=1}^{N}\left(\begin{array}{l}
N \\
n
\end{array}\right)\left(F\left(\gamma_{i}\right)\right)^{N-n}\left(F\left(\gamma_{i+1}\right)-F\left(\gamma_{i}\right)\right)^{n-1} f(x)
$$

for $\gamma_{i}<x<\gamma_{i+1}$ being the quantization intervals, as previously explained.

Through some mathematical simplifications, we can rewrite the PDF of the serving SNR in quantized feedback systems as follows:

$$
f_{Q}(x)=A\left(\gamma_{i}, \gamma_{i+1}\right) f(x)
$$

for $\gamma_{i}<x<\gamma_{i+1}$. where $A\left(\gamma_{i}, \gamma_{i+1}\right)$ is defined as follows:

$$
A\left(\gamma_{i}, \gamma_{i+1}\right)=\frac{F^{N}\left(\gamma_{i+1}\right)-F^{N}\left(\gamma_{i}\right)}{F\left(\gamma_{i+1}\right)-F\left(\gamma_{i}\right)}
$$

Due to the worst case approach followed in power allocation and this new PDF, the transmit power satisfying the QoS demand will increase as follows:

$$
P_{t, q}=\int_{\gamma_{i}}^{\gamma_{i+1}} \frac{q}{\gamma_{i}} f_{Q}(x) \cdot d x
$$

where $\gamma_{i} \leq x<\gamma_{i+1}$. 
The average transmit power over all the quantization intervals, i.e., over all the SNR range, we solve it in a closed form expression as follows:

$$
\bar{P}_{t, q}=\sum_{i=1}^{M} \frac{q}{\gamma_{i}}\left(F_{Q}\left(\gamma_{i+1}\right)-F_{Q}\left(\gamma_{i}\right)\right)
$$

In the same way, we obtain the transmit energy in quantized-feedback systems as:

$$
E_{t, q}=\bar{P}_{t, q}\left(T-\frac{N B}{D_{f}}\right)
$$

In this study, we are interested in the consumed energy per bit as a consumption measure. Hence, the transmit energy should be normalized by the number of transmitted bits. The number of transmitted bits $(\Omega)$ can be easily defined as:

$$
\Omega=D_{t}\left(T-\frac{N B}{D_{f}}\right)
$$

where $D_{t}$ is the data transmission rate, which is equal to the QoS rate. Therefore, the transmit energy per bit in quantized-feedback systems, Equation (15), in [Joule/bit], denoted by $\left(E p B_{t, q}\right)$, is given as:

$$
E p B_{t, q}=\bar{P}_{t, q} \frac{\left(T-\frac{N B}{D_{f}}\right)}{D_{t}\left(T-\frac{N B}{D_{f}}\right)}=\frac{\bar{P}_{t, q}}{D_{t}}
$$

and the transmit energy per bit in non-quantizedfeedback systems, Equation (9), in [Joule/bit], denoted by $\left(E p B_{t, n q}\right)$, is given as:

$$
E p B_{t, n q}=\bar{P}_{t, n q} \frac{\left(T-\frac{N B}{D_{f}}\right)}{D_{t}\left(T-\frac{N B}{D_{f}}\right)}=\frac{\bar{P}_{t, n q}}{D_{t}}
$$

Now, we define the increase in the transmit energy due to the quantization as the additional energy due to quantization $\left(E_{w}\right)$, which represents the difference between the transmit energy per bit in quantized and non-quantized feedback systems as follows:

$$
E_{w}=E p B_{t, q}-E p B_{t, n q}
$$

\subsection{Power outage probability}

Due to multi-path fading and channel fluctuation experienced by users, the scheduled user may have a low SNR, so that the user needs high power to satisfy its QoS. In this section, we deal with the fact that the total available power in the BS is limited, so the scheduled user may experience some power outage [19] when the user needs an amount of power larger than the available power at the transmitter, hence, there will be an outage for the scheduled user. The probability of power outage due to power limitation exists regardless of the feedback type (i.e., quantized or non-quantized).
The power outage occurs when the required transmit power $\left(\frac{q}{x}\right)$ is more than the total power at transmitter $\left(P_{T}\right)$. The probability of power outage $\left(P_{\text {out }}\right)$ is given as:

$$
P_{\text {out }}=\operatorname{Pr}\left\{\frac{q}{x}>P_{T}\right\}=\operatorname{Pr}\left\{x<\frac{q}{P_{T}}\right\}
$$

where $P_{r}$ is the probability symbol. The previous equation translates for the non-quantized feedback system as:

$$
P_{\text {out,nq }}=F_{\max }\left(\frac{q}{P_{T}}\right)
$$

and for quantized feedback systems:

$$
P_{\text {out }, q}=F_{Q}\left(\frac{q}{P_{T}}\right)
$$

where $F_{\max }$ and $F_{Q}$ are the CDFs of $f_{\text {max }}$, given in Equation (5), and $f_{Q}$, given in Equation (11), respectively.

\subsection{Feedback energy}

The system performance will be enhanced by increasing the number feedback bits making the quantized version of the SNR to approach the actual value, which yields a better performance of the opportunistic scheduling, and consequently, less additional energy. On the other hand, the negative effect of increasing the feedback bits is the increase in the required reserved resources, time and energy, for the feedback process, so the users consume a portion of their energy to report the SNR, while the whole system waits additional time until the feedback values are received from all users.

Simply, if we denote the power used in feedback process as $P_{f}$, then for a system with $N$ users where each of them feedbacks its SNR by $B$ bits, the energy consumed during the feedback process $\left(E_{f}\right)$ is given by:

$$
E_{f}=T_{f} P_{f}=\frac{N B}{D_{f}} P_{f}
$$

and the feedback energy per bit, Equation (23), in [Joule/bit], denoted by $\left(E p B_{f}\right)$, is given as:

$$
E p B_{f}=\frac{E_{f}}{D_{t}\left(T-\frac{N B}{D_{f}}\right)}
$$

In order to account for both $E_{w}$ and $E p B_{f}$, an optimization has to be applied to obtain the optimal number of quantization bits that minimizes the overall energy consumption in the system. The optimization problem can be formulated as:

$$
\min _{B} E_{w}+E p B_{f}
$$

where the first term $E_{w}$ is given in Equation (19). Therefore, the problem can be rewritten as follows:

$$
\min _{B} E p B_{t, q}-E p B_{t, n q}+E p B_{f}
$$


It is worthy mentioning that we do not consider the energy consumption during training and synchronization since it is independent of the quantization process. Clearly, the function is non-convex of $B$, and hence, the solution of this problem is infeasible in a closed form. In next section, we solve the problem by computer simulations in order to obtain the optimum number of quantization bits that minimizes the overall energy consumption.

\section{Simulation results}

A multiuser scenario is considered, where the BS intends to communicate with a single user at each scheduling time $T=20 \mathrm{~ms}$. A total of $N=30$ users are available in the system with i.i.d. channel characteristics. A homogeneous system (i.e., all users have the same application) is assumed, where the QoS can be achieved by a data rate of $2 \mathrm{bit} / \mathrm{s} / \mathrm{Hz}$. The average SNR is considered to be SNR $=0 \mathrm{~dB}$, but due to the channel fluctuations, the provided data rate could be lower than the average at some instant. The feedback data rate $\left(D_{f}\right)$ is assumed to be $20 \mathrm{kbps}$. In all figures in this section, the markers represent the simulation results while the solid lines represent the results obtained by the provided equations.

Figure 1 shows the behavior of the transmit energy in both scenarios, quantized, and non-quantized feedback systems, versus the number of quantization bits. The transmit energy for non-quantized feedback system

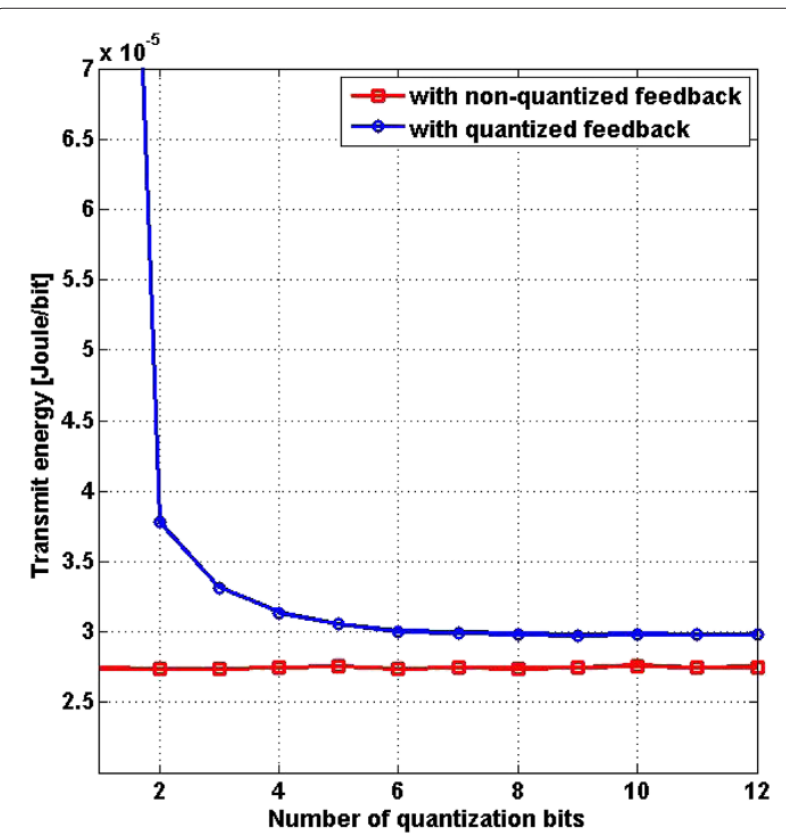

Figure 1 The transmit energy in non-quantized and quantized feedback systems versus the number of quantization bits. (The markers represent the simulation results while the solid lines represent the results obtained by the provided equations). is constant as it is independent of the quantization bits. Related to the quantized feedback scenario, the transmit energy decreases as the number of quantization bits increases. Increasing the quantization bits improves the scheduling procedure toward selecting the actual maximum SNR, leading to less energy consumption. The area between the two curves represents the amount of additional energy due to the quantization, as indicated in Equation (19). It is useful to note that the additional energy is due to, the random selection when more than one user appears as the best user, and the worst case approach followed in the power allocation. Notice that the exact results are obtained by the presented equations in the article.

The exact amount of the additional energy and feedback energy at each number of bits are shown in Figure 2. Notice that as the number of quantization bits increases, the consumed energy during feedback process rises as well. However, While the feedback energy increases, the additional energy decreases as the quantization bits increase, which represent two opposite effects on the overall energy consumption. This motivates the optimization of the number of quantization bits in order to achieve the minimum energy consumption. The power required to feedback the bits $P_{f}$ (the control channel in communication standards) is assumed to be 1 .

The power outage probability is an important parameter to characterize the system. For a variable number of quantization bits the probability of power outage is shown in Figure 3. The probability of power outage decreases

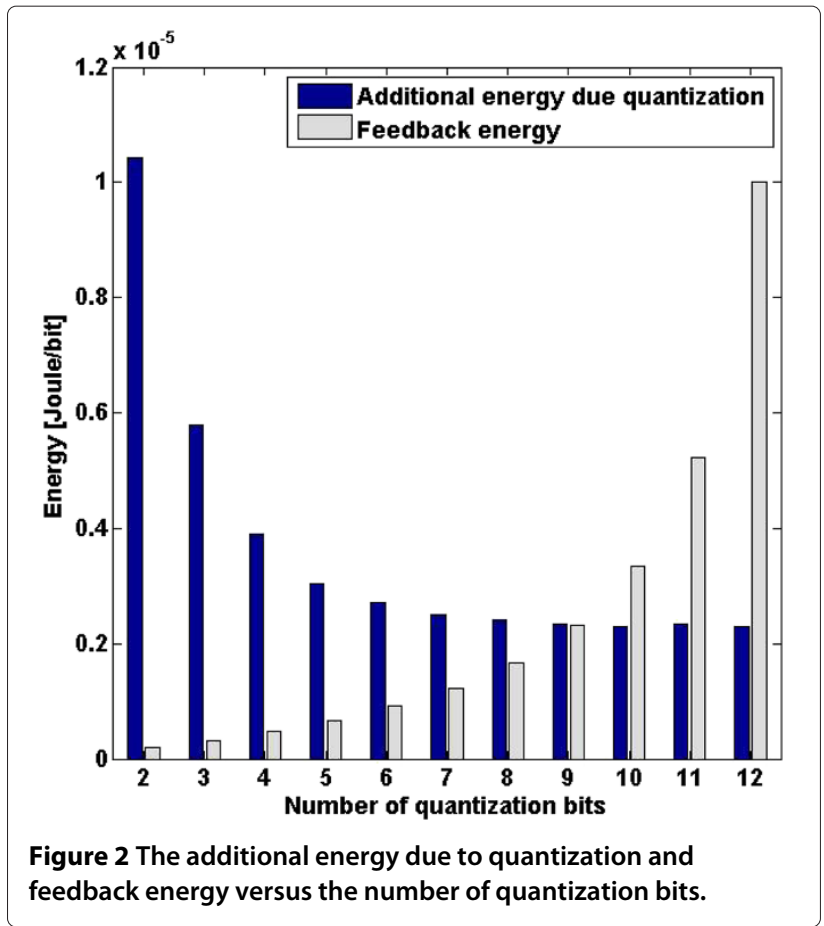




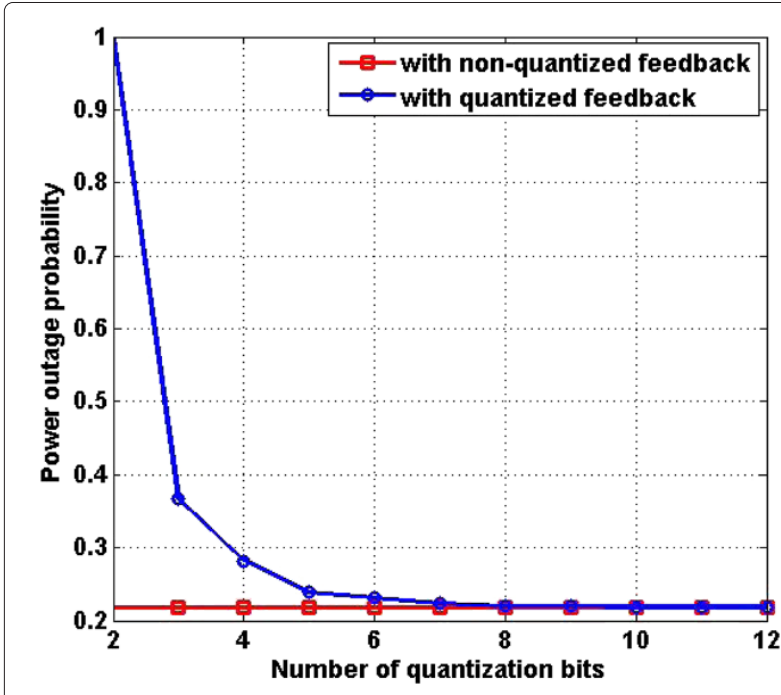

Figure 3 The probability of the power outage in non-quantized feedback and quantized feedback systems versus the number of the quantization bits. (The markers represent the simulation results while the solid lines represent the results obtained by the provided equations). as the number of bits increases, and reaches a constant value which is equal the power outage probability for nonquantized feedback system, as calculated by Equation (21). This decrease is due to approaching the exact value of the scheduled SNR while the number of quantization bits increases, which results in less effect of the worst case assumption on energy required. Both scenarios, quantized and non-quantized, still have power outage probability more than zero because the power resources are limited. Notice the exact matching between simulation and analytical results.

We have concluded from the previous section that there is an optimum number of the quantization bits that will yield in the minimum energy consumption. We also formulated the energy minimization problem which was not mathematically solved in a closed form. Figure 4 shows the energy consumption of the system versus the number of quantization bits. We can see the that the consumed energy for low number of bits (less than 5 bits) is high due to the large additional energy caused by quantization. In contrast, for higher number of bits (more than 7), the large energy consumption is due to the energy consumed in the feedback process. Figure 4 also shows that at the range of 5-7 bits the energy consumption is relatively small and reaches its minimum value when the number of bits is 6 .

In order to clarify the effect of the feedback power, Figure 5 represents a 3D graph of the consumed energy versus the number of quantization bits and the feedback power. Notice that the optimal number of bits decreases as the feedback power increases. This can be justified

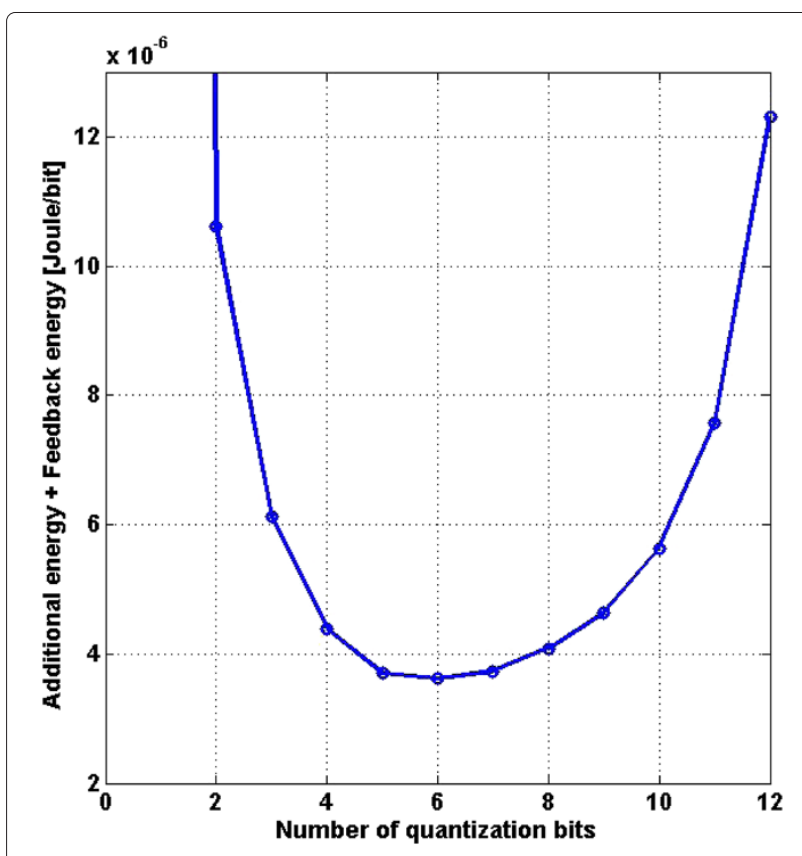

Figure 4 The sum of additional energy and feedback energy versus the number of quantization bits.

as follows, as the feedback power increases, the effect of the feedback energy on the overall energy consumption increases, which means a need for a less number of bits that reduces the overall energy consumption.

\section{Conclusions}

In this article, we present a deep analysis on the quantization effects on the energy resources, where we obtain a closed form expression for the transmit energy in quantized and non-quantized feedback systems, the power outage probability, and the additional energy due to quantization.

In addition, we formulate an optimization problem, that aids to find the optimum number of quantization bits that minimizes the overall energy consumption.

The obtained equations for each effect are checked by simulation, where the results show a very tight agreement with the mathematical formulation. As a future work, the optimization problem could be reformulated for systems with multiple antenna and variable transmission rates.

\section{Appendix}

\section{Derivation of Equation (8)}

Using Equation (5), we can rewrite Equation (4) as:

$$
\bar{P}_{t, n q}=\int_{0}^{\infty} \frac{q}{x} N \sigma^{2} e^{-\sigma^{2} x}\left(1-e^{-\sigma^{2} x}\right)^{N-1} d x
$$




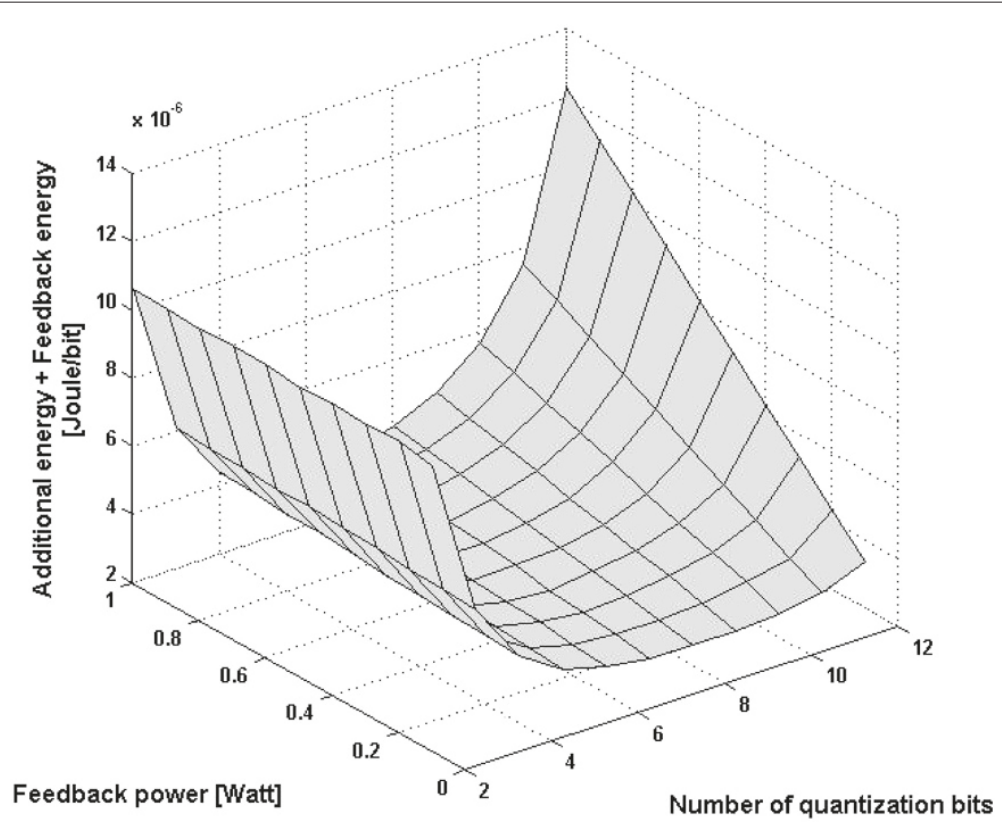

Figure 5 The sum of additional energy and feedback energy versus the number of quantization bits and the feedback power.

Using the binomial power series, we write:

$$
\bar{P}_{t, n q}=\int_{0}^{\infty} \frac{q}{x} N \sigma^{2} \sum_{n=0}^{N-1}\left(\begin{array}{c}
N-1 \\
n
\end{array}\right)(-1)^{n} e^{-\sigma^{2} x(n+1)} d x
$$

which can be rearranged as:

$$
\bar{P}_{t, n q}=N \sigma^{2} q \sum_{n=0}^{N-1}\left(\begin{array}{c}
N-1 \\
n
\end{array}\right)(-1)^{n} \int_{0}^{\infty} \frac{e^{-\sigma^{2} x(n+1)}}{x} d x
$$

The integral can be divided into two integrals as follows:

$$
\begin{aligned}
\bar{P}_{t, n q}= & N \sigma^{2} q \sum_{n=0}^{N-1}\left(\begin{array}{c}
N-1 \\
n
\end{array}\right)(-1)^{n} \\
& \times\left(\int_{0}^{1} \frac{e^{-\sigma^{2} x(n+1)}}{x} d x+\int_{1}^{\infty} \frac{e^{-\sigma^{2} x(n+1)}}{x} d x\right)
\end{aligned}
$$

where the second integral can be replaced by the exponential integral, defined as $E_{1}(x)=\int_{1}^{\infty} \frac{e^{-x t}}{t} \cdot d t[20]$, as follows:

$$
\begin{aligned}
\bar{P}_{t, n q}= & N \sigma^{2} q \sum_{n=0}^{N-1}\left(\begin{array}{c}
N-1 \\
n
\end{array}\right)(-1)^{n} \\
& \times\left(\int_{0}^{1} \frac{e^{-\sigma^{2} x(n+1)}}{x} d x+E_{1}\left(\sigma^{2}(n+1)\right)\right)
\end{aligned}
$$

It remains to solve the first integral which is an improper integral. Using the power series representation of the exponential function, the integral is written as follows:

$$
\begin{aligned}
\bar{P}_{t, n q}= & N \sigma^{2} q \sum_{n=0}^{N-1}\left(\begin{array}{c}
N-1 \\
n
\end{array}\right)(-1)^{n} \\
& \times\left(\int_{0}^{1} \sum_{i=0}^{\infty} \frac{\left(-\sigma^{2}(n+1) x\right)^{i}}{i ! x} d x+E_{1}\left(\sigma^{2}(n+1)\right)\right)
\end{aligned}
$$

that is simplified as

$$
\begin{aligned}
\bar{P}_{t, n q}= & N \sigma^{2} q \sum_{n=0}^{N-1}\left(\begin{array}{c}
N-1 \\
n
\end{array}\right)(-1)^{n} \\
& \times\left(\int_{0}^{1} \sum_{i=0}^{\infty} \frac{\left(-\sigma^{2}(n+1)\right)^{i}(x)^{i-1}}{i !} d x+E_{1}\left(\sigma^{2}(n+1)\right)\right)
\end{aligned}
$$

The first term $(i=0)$ in the third summation is constant. Therefore, by applying the identity $\sum_{h=0}^{H}\left(\begin{array}{c}H \\ h\end{array}\right)(-1)^{h} a=$ 0 when $a=$ constant on this term and the second summation, the last equation becomes as:

$$
\begin{aligned}
\bar{P}_{t, n q}= & N \sigma^{2} q \sum_{n=0}^{N-1}\left(\begin{array}{c}
N-1 \\
n
\end{array}\right)(-1)^{n} \\
& \times\left(\int_{0}^{1} \sum_{i=1}^{\infty} \frac{\left(-\sigma^{2}(n+1)\right)^{i} x^{i-1}}{i !} d x+E_{1}\left(\sigma^{2}(n+1)\right)\right)
\end{aligned}
$$


Then, by performing the integration, we get

$$
\begin{aligned}
\bar{P}_{t, n q}= & N \sigma^{2} q \sum_{n=0}^{N-1}\left(\begin{array}{c}
N-1 \\
n
\end{array}\right)(-1)^{n} \\
& \times\left(\sum_{i=1}^{\infty} \frac{\left(-\sigma^{2}(n+1)\right)^{i}}{i \cdot i !}+E_{1}\left(\sigma^{2}(n+1)\right)\right)
\end{aligned}
$$

The last summation can be evaluated as follows [20]:

$$
\begin{aligned}
\bar{P}_{t, n q}= & N \sigma^{2} q \sum_{n=0}^{N-1}\left(\begin{array}{c}
N-1 \\
n
\end{array}\right)(-1)^{n} \\
\times & \left(-\delta-\ln \left(\sigma^{2}(n+1)\right)-E_{1}\left(\sigma^{2}(n+1)\right)\right. \\
& \left.+E_{1}\left(\sigma^{2}(n+1)\right)\right)
\end{aligned}
$$

where $\delta$ is Euler constant [20]. Using the previous identity as $\delta$ is a constant, the amount of required transmit power is given as:

$$
\bar{P}_{t, n q}=-N \sigma^{2} q \sum_{n=0}^{N-1}\left(\begin{array}{c}
N-1 \\
n
\end{array}\right)(-1)^{n+1}\left(\ln \left(\sigma^{2}(n+1)\right)\right)
$$

as given in Equation (8).

\section{Endnotes}

${ }^{a} \mathrm{~A}$ single cell is tackled, and no interference from other cells is considered. The article's contribution is not targeted to cellular networks but to the quantization and energy efficiency.

${ }^{b}$ Along the article, all the users are assumed to have the same average channel characteristics, and showing the same distribution for the channel and SNR value. If this is not the case (e.g., heterogeneous users distribution in the cell, with some users far from the BS), then a channel normalization (e.g., division by the path loss) can be accomplished for such a scenario. This is done because the article's contribution is independent of the path loss.

\section{Competing interests}

The authors declare that they have no competing interests.

\section{Acknowledgements}

This study was funded by the Research Projects GREENET (PITN-GA-2010-264759), CO2GREEN (TEC2010-20823), and GREEN-T (TSI-020400-2011-16-CP8-006).

\section{Author details}

${ }^{1}$ University of Trento, Trento, Italy. ${ }^{2}$ Qatar Mobility Innovations Center (QMIC), AlDoha, Qatar. ${ }^{3}$ Telecommunications Technological Centre of Catalonia, Barcelona, Spain.

Received: 20 December 2012 Accepted: 27 February 2013

Published: 21 March 2013

\section{References}

1. G Fettweis, E Zimmermann, in Proceedings of the 11th International Symposium on Wireless Personal Multimedia Communications. ICT energy consumption-trends and challenges, (Lapland, Finland, 2008), p. 6
2. D Love, R Heath, V Lau, D Gesbert, BD Rao, M Andrews, An overview of limited feedback in wireless communication systems. IEEE J. Sel. Areas Commun. 26(8), 1341-1365 (2008)

3. RM Gray, DL Neuhoff, Quantization. IEEE Trans. Inf. Theory. 44(6), 2325-2383 (1998)

4. J Proakis, Digital Communications, 4th edn. (McGraw-Hill, New York, 2000)

5. Y Isukapalli, BD Rao, Packet error probability of a transmit beamforming system with imperfect feedback. IEEE Trans. Signal Process. 58(4), 2298-2314 (2010)

6. F Floren, O Edfors, BA Molin, in Proceedings of IEEE Global Telecommunications Conference. The effect of feedback quantization on the throughput of a multiuser diversity scheme, (San Francisco, USA, 2003), pp. 497-501

7. O Ozdemir, M Torlak, Optimum feedback quantization in an opportunistic beamforming scheme. IEEE Trans. Wirel. Commun. 9(5), 1584-1593 (2010)

8. CR Murthy, BD Rao, Quantization methods for equal gain transmission with finite rate feedback. IEEE Trans. Signal Process. 55(1), 233-245 (2007)

9. A Lau, FR Kschischang, Feedback quantization strategies for multiuser diversity systems. IEEE Trans. Inf. Theory. 53(4), 1386-1400 (2007)

10. ED Wardihani, W Wirawan, G Hendrantoro, in Proceedings of the International Conference In Advanced Computer Science and Information System (ICACSIS). Optimal quantization and energy allocation schemes in distributed estimation for wireless sensor networks, (Surabaya, Indonesia, 2011), pp. 57-62

11. X Luo, GB Giannakis, in Proceedings of First Annual IEEE Communications Society Conference on Sensor and Ad Hoc Communications and Networks (SECON). Energy-constrained optimal quantization for wireless sensor networks, (Snata Clara, Canada, 2004), pp. 272-278

12. AG Marques, ABR Gonzalez, JL Rojo-Alvarez, J Requena-Carrion, J Ramos, Optimizing average performance of OFDM systems using limited-rate feedback. IEEE Trans. Wirel. Commun. 9(10), 3130-3143 (2010)

13. O Biggelaar, JM Dricot, PD Doncker, F Horlin, in Proceedings of IEEE Vehicular Technology Conference-Spring. Quantization and transmission of the energy measures for cooperative spectrum sensing, (Brussels, Belgium, 2010), pp. 1-5

14. A Khoshnevis, A Sabharwal, On the asymptotic performance of multiple antenna channels with quantized feedback. IEEE Trans. Wirel. Commun. 7(10), 3869-3877 (2008)

15. P Viswanath, DN Tse, R Laroia, Opportunistic beamforming using dumb antennas. IEEE Trans. Inf. Theory. 48(6), 1277-1294 (2002)

16. P Svedman, SK Wilson, LJ Cimini, B Ottersten, Opportunistic beamforming and scheduling for OFDMA systems. IEEE Trans. Commun. 55(5), 941-952 (2007)

17. L Yang, MS Alouini, Performance analysis of multiuser selection diversity. IEEE Trans. Veh. Technol. 55(6), 1848-1861 (2006)

18. N Zorba, Al Perez-Neira, Robust power allocation schemes for multibeam opportunistic transmission strategies under quality of service constraints. IEEE J. Sel. Areas Commun. 26(6), 1025-1034 (2008)

19. S Kandukuri, S Boyd, Optimal power control in interference-limited fading wireless channels with outage-probability specifications. IEEE Trans. Wirel. Commun. 1(1), 46-55 (2002)

20. IS Gradshteyn, IM Ryzhik, Table of Integrals, Series, and Products, 4th edn. (Academic Press, New York, 1994)

doi:10.1186/1687-1499-2013-83

Cite this article as: Althunibat et al:: Energy optimization in multiuser quantized feedback systems. EURASIP Journal on Wireless Communications and Networking 2013 2013:83. 